$\underline{\text { Iranian Journal of Pathology | ISSN: 2345-3656 }}$

\title{
Cervical Cancer and Genital Infections: Assessment of Performance and Validation in Human Papillomavirus Genotyping Assays in Iran, its Neighbouring Countries and Persian Gulf Area
}

\begin{tabular}{|c|c|}
\hline 1. Dep & $\begin{array}{l}\text { Amir Sohrabi', Masoud Hajia*1 } \\
\text { olecular Biology, Research Center of Health Reference Laboratory, } \\
\text { Ministry of Health and Medical Education, Tehran, Iran }\end{array}$ \\
\hline KEYWORDS & ABSTRACT \\
\hline $\begin{array}{l}\text { HPV } \\
\text { Cervical Cancer } \\
\text { Genital Infection } \\
\text { Genotyping Assay } \\
\text { Iran } \\
\text { Persian Gulf }\end{array}$ & $\begin{array}{l}\text { Background: The accuracy of diagnostic assays in Human Papillomavirus (HPV) } \\
\text { genital infection and cervical cancer has remained a clinical challenge in diagnosis. } \\
\text { Evidence indicates that a large proportion of cervical cancer can be prevented } \\
\text { through organized care for HPV and testing. Countries with low per capita income, } \\
\text { such as Iran and its neighbours, have no national organized program for cervical } \\
\text { cancer screening and vaccination. The aim of this study was to review recent } \\
\text { published papers in this region for evaluating the efficacy of released data regarding } \\
\text { HPV genotyping system in genital infections and cervical cancer } \\
\text { Methods: Investigating various medical search engines retrieved } 46 \text { reports, } \\
\text { mostly after 2010, consisting of either home brew protocols or commercial } \\
\text { technologies in this field. } \\
\text { Results: Summarized results demonstrated that except a few cases, all reports } \\
\text { were limited studies performed in confined populations focusing on attending }\end{array}$ \\
\hline Article Info & $\begin{array}{l}\text { patients at clinics for regular checkups. In the present study, } 52.8 \% \text { of papers were } \\
\text { from Iran and the rest belonged to other countries. The rate of HPV infection was } \\
\text { reported in the range of } 0.62 \% \text { to } 25 \% \text { in the normal population, while it varied from } \\
18.75 \% \text { to } 100 \% \text { in females with cervical cancer. In HPV genotyping surveys, only } \\
26.1 \%(12 / 46) \text { of reports had validated and World Health Organization (WHO) }\end{array}$ \\
\hline $\begin{array}{l}\text { Received 2016-Apr-03 } \\
\text { Accepted 2016-Aug-25 }\end{array}$ & $\begin{array}{l}\text { proficient procedures. Also, multiple infections were not mentioned in } 56.52 \% \\
(25 / 46) \text { of researches. }\end{array}$ \\
\hline Published Online 27 Jan 2017; & $\begin{array}{l}\text { Conclusions: Employing reliable genotyping methods is the best way for regular } \\
\text { screening of cervical cancer related to HPV and precancerous diseases in females of } \\
\text { these areas. The focus of most surveys was to come up with the best national policies } \\
\text { for establishing a preventive program in Iran and Persian Gulf area. }\end{array}$ \\
\hline
\end{tabular}

Corresponding Information: Dr. Masoud Hajia, Dept. of Molecular Biology, Research Center of Health Reference Laboratory, Ministry of Health and Medical Education, Tehran, Iran. Tel: +982166728112 Email: massoudhajia@yahoo.com

Copyright ( 9 2016, IRANIAN JOURNAL OF PATHOLOGY. This is an open-access article distributed under the terms of the Creative Commons Attribution-noncommercial 4.0 International License which permits copy and redistribute the material just in noncommercial usages, provided the original work is properly cited.

\section{Introduction}

More than 200 Human Papillomavirus (HPV) genotypes have been detected, approximately 40 of which can infect the genital tract. Infection with highrisk HPV is considered a major cause of cervical cancer $(1,2)$. Based on WHO guidelines, approximately sixteen HPV genotypes have been diagnosed in laboratories (HPVs 6,11 [Low Risks], 16,18,31, 33,35,39,45,51,52,56,58,59,66,68 [High
Risks]). Human Papillomavirus genotyping test refers to HPV IVD devices, which detect and should be able to differentiate between high-risk and low-risk HPV genotypes (3, 4). Al-Awadhi et al. 2011, underlined that most females with normal cytology could be infected with low and high-risk genotypes (5). Several commercial technologies are available such as Digene Hybrid Capture 2 High-Risk HPV DNA 
Test, Cervista $^{\mathrm{TM}}$ HPV HR and Genfind ${ }^{\mathrm{TM}}$ DNA Extraction Kit, Cervista $^{\mathrm{TM}}$ HPV 16/18, Cobas HPV Test and APTIMA ${ }^{\circledR}$ HPV Assay, which are Food and Drug Administration (FDA) approved (4,6). However, improving the efficacy of the diagnostic methods for HPV detection and genotyping assays is still one of the main objectives in this field. It seems that DNA-based methods for HPV detection and genotyping are mostly designed for L1, E6/E7, E1 and E2 of HPV genome. These regions are more sensitive and specific than others. In most diagnostic commercial assays, the $\mathrm{L} 1$ region is used. The L1 region has conserved and variable sequences, therefore it is suitable for HPV detection, genotyping and classification (6-9). Abru et al. have reviewed the current HPV genotyping methods. They underlined that despite several methods being available and some molecular techniques being developed, HPV protocols still need to become more rapid, automated, and cost effective, to be of practical use in low income countries (7). Besides an assessment of the regional distribution of HPV genotypes and accurate evaluation of human papillomavirus infections, genotyping is still of critical importance to evaluate the epidemiology of HPV infections worldwide and to establish and monitor HPV vaccine efficacy. Reporting true negative results has an important role in decreasing the prevalence of the HPV. It is important to note that negative results of HPV DNA may not negate HPV infection, and failure of HPV diagnostic methods and incorrectly interpreted results may lead to incorrect treatment and cervical cancer related to HPV screening (10).

The cost of these assays effects the selection of the HPV genotyping methods in both developing and under developed countries.

Discrimination between the high and low risk genotypes of HPV in clinical diagnostic exposures is one of the concerns in developing countries, and countries that do not have access to new technologies (7-9). This study reviewed researches related to Iran's neighbouring countries and the Persian Gulf area, which had focused on HPV genotyping technologies and prevalence of HPVs in single and multiple genital infections and cervical cancer.

\section{Materials and Methods}

\section{Study Design}

This Meta-analysis focused on recent HPV reports (2010 to 2015) in our region. This review included all researches on genital infections and cervical cancer related to HPV from Iran, Pakistan, Afghanistan, Iraq, Kuwait, Saudi Arabia, Bahrain, Qatar, Oman, Yemen, Jordan and Syria. Northern neighbouring countries of Iran were excluded from this study, as they belong to Russian Federation countries (the western countries of the former Soviet Union, Caucasus Region and central Asia).

\section{Data Sources}

We searched several relevant international medical databases including PubMed, Scopus, and Google Scholar, as well as local databases. We also checked the reference lists of retrieved articles to identify additional studies.

\section{Data Extraction}

The data were extracted and summarized. The final eligible articles were reviewed by both authors and disagreements were resolved by consensus. The following study characteristics were extracted, first author, year of publication, region of the study, studied group, detection method, and analyzed results. The applied method was assessed by accepted international approved guidelines and protocols such as WHO and Food and Drug Administration (FDA). Papers with no accurate method or results were excluded from the review. Necessary items for qualified technique were as follows:

\section{Sample Preparation Methods}

Application of a proper method for specimen collection and shipment had critical roles in HPV results. Cellular samples from the site of infection and cervical transformation zone are the best specimens for molecular diagnosis in both cervical cancer and genital infections. We should be aware that suitable specimens are those with infected cells such as Liquid Based Cytology specimens (LBCs), Formalin Fixed Paraffin Embedded Tissue (FFPE), or a portion of genital warts and lesions. Those specimens stored at room temperature ( $\sim 14$ days) or at $4^{\circ} \mathrm{C}$ for $\sim \geq$ three weeks are not recommended for testing. The desired storage condition is $-20^{\circ} \mathrm{C}$ for long durations $(3,4)$. 


\section{Home Brew Human Papilloma Protocols}

The clinical performance of a qualitative test (test with two outcomes, positive or negative) should be described by its clinical sensitivity and specificity. The results of home brew protocols are required to be established in prospective clinical studies, since the results are going to be considered as final decisions for HPV infections. All effective criteria for true results should be evaluated and determined according to the FDA and WHO guidelines. At present, PGMY, GP5/GP6, MY09/MY11 and SPF10 general primers are used in most commercial and home-brew diagnostic systems, which are validated and utilized by WHO and companies as screening methods. These primer pairs amplify part of the L1 HPV genome as L1 consensus Polymerase Chain Reaction (PCR). The rest of primers, which are used in research projects and clinical laboratories, should be qualified and participated in proficiency study and quality assessment programs.

\section{Differentiation of True Negative from False Negative}

Reporting false negative and positive results could lead to missing proper treatment strategies and increasing morbidity and mortality of cervical cancer. Applying an Internal Control (IC) is considered to be mandatory for diagnosis. False positive results may be due to contamination, carry over and sampling cross contamination. False negative results could be affected by low virus copy number, sampling and specimen collection errors. The use of Oral Contraceptive Pills (OCPs), topical ointments, cryotherapy and laser therapy could be regarded as inhibitory for amplification and may interfere with DNA-based methods.

\section{Low and High Risk Types}

It has been frequently reported that low risk types have a considerable role in genial infections and even cervical cancer with low percentage. These low risk types, especially HPVs 6 and 11, are relatively common in atypical squamous cells of undermined significance (ASCUS), although can play a role in mixed infections. Therefore, detection of dominant genotypes must be included in any diagnostic protocol (11). Fourteen HPV high-risk types $(16,18,31,33,35,39,45,51,52,56,58,59,66,68) \quad$ plus HPV 6 and 11 are included in proficiency panel testing of WHO.

\section{The World Health Organization Human Papillomavirus LabNet}

The WHO in collaboration with the Karolinska Institute in Sweden has established the HPV LabNet. Evaluation on performance of HPV diagnostic methods, during annual proficiency testing program, is performed, especially in Reference Health Laboratories around the world. Commercial and inhouse diagnostic assays for HPV genotyping are evaluated. Results of this program are the best reference for better understanding of HPV genotyping methods performance. Hence, in the present study, based on these results, efficiency, validation and comparison of HPV genotyping technologies are appraised.

\section{Results}

Various search engines revealed 46 reports, performed mostly after 2010. The number of applied commercial kits or home brew protocols was different in each area of our region (Table 1 and 2). Valid general primers such as GP5/6 and MY09/MY11 for general HPV detection were used in $41.3 \%$ (19/46) of published articles and $58.7 \%$ (27/46) used other sequences. It seems that most procedures for HPV genotyping methods were not mentioned in $\mathrm{WHO}$ and FDA guidelines in these areas. We considered $\mathrm{HC} 2$, LiPA, Roche, and GenoArray commercial diagnostic kits as approved genotyping methods. Other used genotyping methods were unconfirmed protocols or did not fully cover all necessary types such as some PCR hybridization methods, PCR sequencing, multiplex PCR, and Real Time PCR method. Only one home-brew HPV genotyping method, In-House Multiplex Real time PCR, was approved in WHO proficiency testing among these reports (12). Overall, 13 out of 46 papers used protocols covering all necessary detected types consisting of 10 approved methods, 2 commercially unapproved procedures and one home brew developed method (Table 1 and 2). Studied population reports were based on patients attending clinics for regularly checkups, cervical cancer, abnormal cytology, negative pap smears, genital wart, and HPV positive samples were found in $16,14,3,1,1$ and 1 reports, respectively. Ten papers had established their investigation on a mixed source of specimens. The prevalence of HPV in patients, who attended for routine checkups varied from $0.62 \%$ to $25 \%$ in performed researches. Those reports on 
specimens collected from patients with cervical cancer specimens also had variable prevalence from as low as $18.75 \%$ to $90.76 \%$ and even $100 \%$. Multiple infections were recorded in some articles; 20 reports
(63.04\%) out of 46 investigates, 10 from Iran and 10 from Arabic countries but none from Pakistan. Our search retrieved no data from Jordan, Oman and Afghanistan in scientific medical databases.

Table 1: Human Papillomavirus Genotypes Distribution in Genital Infection and Cervical Cancer in Iran

\begin{tabular}{|c|c|c|c|c|}
\hline Author/Year & Region & HPV Detection Method & Number /Type of Study & HPV Positive/ HPV Genotypes /Co infection \\
\hline $\begin{array}{l}\text { Hamkar et al., } 2002 \\
\text { [13] }\end{array}$ & $\begin{array}{l}\text { Mazandaran/ } \\
\text { Iran }\end{array}$ & Home-brew PCR & $\begin{array}{l}\text { 100/ Routine Checkups \& } \\
\text { Cervical Cancer }\end{array}$ & $\begin{array}{l}\text { 78.6\% / HPV 16,18,31,33,6,11/ HPV16/18: } 1.51 \% \text {, } \\
\text { HPV31/33: 16.24\%, HPV6/11: 4.18\% }\end{array}$ \\
\hline $\begin{array}{l}\text { Mortazavi et al., } 2002 \\
\text { [14] }\end{array}$ & Tehran/Irn & $\begin{array}{lrr}\text { In Situ Hybridization \& } & \text { Home } \\
\text { Brew PCR (Triplex } & \text { PCR } \\
16,18,33 \text { ) } & & \\
\end{array}$ & $\begin{array}{l}\text { 100/ FFPE Cervical } \\
\text { Cancer }\end{array}$ & $82 \%$ / HPV 16,18,3 / Not Mentioned \\
\hline $\begin{array}{l}\text { Hamkar et al., } 2003 \\
\text { [15] }\end{array}$ & $\begin{array}{l}\text { Mazandaran/ } \\
\text { Iran }\end{array}$ & $\begin{array}{l}\text { Home-brew PCR \& In Situ } \\
\text { Hybridization }\end{array}$ & $\begin{array}{l}\text { 254/ Routine checkups \& } \\
\text { Cervical Cancer }\end{array}$ & 44.48\% / HPV 16,18,6,11,31,33/ Not Mentioned \\
\hline $\begin{array}{l}\text { Farjadian et al.,2003 } \\
\text { [16] }\end{array}$ & Shiraz/Iran & $\begin{array}{l}\text { Home-brew PCR } \\
\text { ( Duplex PCR 16\&18) }\end{array}$ & $\begin{array}{l}\text { 101/ FFPE Cervical } \\
\text { Cancer }\end{array}$ & $87.12 \%$ / HPV16,18 / Not Mentioned \\
\hline Zandi et al., 2010 [17] & Bushehr/ Iran & $\begin{array}{l}\text { Home Brew PCR GP5/GP6 \& } \\
\text { Sequencing }\end{array}$ & 200/ Routine Checkups & $5.5 \%$ / HPV 16,18,53 / Not Mentioned \\
\hline Safaie et al., 2010 [18] & Shiraz/Iran & $\begin{array}{l}\text { Home-brew PCR } \\
\text { ( Duplex PCR 16\&18) }\end{array}$ & 402/ Routine Checkups & $5.47 \%$ / HPV 16 / Not Mentioned \\
\hline $\begin{array}{l}\text { Shahramian et al., } \\
2011[19]\end{array}$ & Zabol/ Iran & $\begin{array}{l}\text { Home Brew PCR } \\
\text { GP5/GP6 - MY09/MY11 \& } \\
\text { Duplex PCR 16\&18 }\end{array}$ & 402/ Routine Checkups & $21.39 \%$ / HPV 16,18 / Not Mentioned \\
\hline $\begin{array}{l}\text { Jaberipour et al.,2011 } \\
{[20]}\end{array}$ & Shiraz/Iran & $\begin{array}{l}\text { Real-time for } 16,18,33,52 \\
\text { (Primer Design®) }\end{array}$ & $\begin{array}{l}\text { 100/ Tissue, Biopsy, } \\
\text { Blood \& Genital Wart }\end{array}$ & $19 \% / \mathrm{HPV} 16,18 / 2 \%$ \\
\hline $\begin{array}{l}\text { Jaberipour et al.,2011 } \\
\text { [21] }\end{array}$ & Shiraz/ Iran & $\begin{array}{l}\text { Real-time for } 16,18,33,52 \\
\text { (Primer Design®) }\end{array}$ & 79/ Genital Wart & $16.45 \%$ / HPV 16,18 / $1.3 \%$ \\
\hline $\begin{array}{l}\text { Moradi et al.,2011 } \\
\text { [22] }\end{array}$ & Gorgan/ Iran & $\begin{array}{l}\text { Home Brew PCR } \text { GP5/GP6- } \\
\text { MY09/MY11 \& } \\
\text { Duplex PCR } 16 \& 18\end{array}$ & 308/ Routine Checkups & $24.67 \%$ / HPV 16,18 / Not Mentioned \\
\hline $\begin{array}{l}\text { Allameh et al.,2011 } \\
\text { [23] }\end{array}$ & Isfahan/ Iran & $\begin{array}{l}\text { Home Brew PCR GP5/GP6 \& } \\
\text { Primer Specific PCR HPV } \\
6,11,16,18\end{array}$ & 130/ Abnormal Cytology & $24.67 \%$ / HPV 16,18,6,11 / $83.1 \%$ \\
\hline $\begin{array}{l}\text { Shahsia et al.,2011 } \\
\text { [24] }\end{array}$ & Tehran/Iran & $\begin{array}{l}\text { Home Brew PCR GP5/GP6 \& } \\
\text { Sequencing }\end{array}$ & 87/ FFPE Cervical Cancer & 79.31\% / HPV 16,18,31,45/ Not Mentioned \\
\hline $\begin{array}{ll}\text { Khodakarami } & \text { et } \\
\text { al.,2011 [25] }\end{array}$ & Tehran/Iran & Home Brew PCR GP5/GP6 & $\begin{array}{l}\text { 825/ Normal \& Abnormal } \\
\text { Cytology }\end{array}$ & $\begin{array}{l}7.75 \% \text { / HPV } 16,18,31,39,45,51,52,56,58,59, \\
73,6,32,40,42,5466,67 / \geq 3 \%\end{array}$ \\
\hline $\begin{array}{l}\text { Eghbali et al.,2012 } \\
\text { [26] }\end{array}$ & Bushehr/Iran & $\begin{array}{l}\text { Home Brew PCR GP5/GP6 \& } \\
\text { LiPA }\end{array}$ & 799/ Routine Checkups & $0.62 \%$ / HPV $16,18,31,33,51,56,66 / 0.25 \%$ \\
\hline $\begin{array}{l}\text { Hamidifard et al.,2012 } \\
\text { [27] }\end{array}$ & Ahvaz/Iran & $\begin{array}{l}\text { Home Brew PCR GP5/GP6 \& } \\
\text { Sequencing }\end{array}$ & $\begin{array}{lll}60 / & \text { FFPE } & \text { (Normal \& } \\
\text { Abnormal) } & & \\
\end{array}$ & $13.33 \%$ / HPV 16,18,6,11/ Not Mentioned \\
\hline $\begin{array}{l}\text { Moeini } \\
\text { [28] }\end{array}$ & Tehran/Iran & Home Brew PCR MY09/MY11 & 96 / FFPE Cervical Cancer & 18.75\% / HPV Positive/ Not Mentioned \\
\hline $\begin{array}{l}\text { Haghshenas et al., } \\
\text { 2013[29] }\end{array}$ & Sari/ Iran & Amplisens ${ }^{\circledR}$ & 98/ FFPE Cervical Cancer & 79.59\% / HPV 16,18,45,39 / Not Mentioned \\
\hline $\begin{array}{l}\text { Shafaghi et al., } 2013 \\
\text { [30] }\end{array}$ & Tehran/Iran & $\begin{array}{lccr}\text { Home } \quad \text { Brew } & \text { Nested } & \text { PCR } \\
\text { MY09/MY11, } & \text { GP5/GP6 } & \& \\
\text { RFLP } & & & \end{array}$ & 851/ Routine Checkups & $\begin{array}{l}30.08 \% \\
\text { HPV16,18,31,33,35,39,45,51,52,56,58,59,66,68,6,11, } \\
42,43,44 / 29.1 \%\end{array}$ \\
\hline $\begin{array}{ll}\text { Mobinikeshe } & \text { et } \\
\text { al.,2013 [31] }\end{array}$ & $\begin{array}{l}11 \text { Province/ } \\
\text { Iran }\end{array}$ & $\begin{array}{l}\text { Home Brew Nested PCR } \\
\text { Only HPV } 16\end{array}$ & 108/ Routine Checkups & $25 \%$ / HPV 16 / Not Mentioned \\
\hline $\begin{array}{l}\text { Shanehsazzadeh et al., } \\
2014 \text { [32] }\end{array}$ & Isfahan/Iran & $\begin{array}{l}\text { Home Brew PCR GP5/GP6 \& } \\
\text { Duplex PCR } 16 \& 18\end{array}$ & $\begin{array}{lll}156 / & \text { FFPE } & \text { Cervical } \\
\text { Cancer }\end{array}$ & $58.97 \%$ / HPV 16,18 / $3.85 \%$ \\
\hline $\begin{array}{l}\text { Pouryasin et al., } 2014 \\
\text { [33] }\end{array}$ & Tehran/ Iran & LiPA & 80/ HPV Positive Patients & $\begin{array}{l}100 \% / 6,16,53,18,52,39,66,11,31,33,35,45,56,58, \\
68,82,54,44,69 / 71 / \text { Co-infection } 50 \% \\
2 \text { types : } 31.1 \%, 3 \text { types: } 8.7 \%, 4 \text { type: } 6.3 \%, \\
5 \text { types : } 2.5 \%, 6 \text { type: } 1.2 \%\end{array}$ \\
\hline $\begin{array}{l}\text { Mirzaei-Kashani et } \\
\text { al., 2014 [34] }\end{array}$ & Isfahan/ Iran & 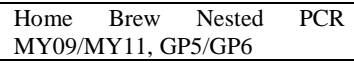 & $\begin{array}{lll}122 / & \text { FFPE } & \text { Cervical } \\
\text { Cancer }\end{array}$ & 76.39\% / HPV Positive / Not Mentioned \\
\hline $\begin{array}{l}\text { Sohrabi et al., } 2014 \\
{[12]}\end{array}$ & Tehran/Iran & $\begin{array}{l}\text { Home Brew TaqMan Real Time } \\
\text { PCR }\end{array}$ & $\begin{array}{l}\text { 112/ Routine Checkups \& } \\
\text { Cervical Cancer }\end{array}$ & $\begin{array}{l}93.75 \% \text { / HPV } 6,11,16,18,31,33,35,39,45,51,52,56, \\
58,59,68 / \leq 12.4 \%\end{array}$ \\
\hline $\begin{array}{l}\text { Mohesni-Mehran et } \\
\text { al., 2015 [35] }\end{array}$ & Rasht/ Iran & Home Brew PCR GP5/GP6 & $\begin{array}{l}\text { 103/ Routine Checkups \& } \\
\text { Abnormal Cytology }\end{array}$ & $4.9 \% /$ HPV Positive/ Not Mentioned \\
\hline
\end{tabular}


Table 2. Human Papillomavirus Genotypes Distribution in Genital Infection and Cervical Cancer in Iran's Neighbouring Countries and Persian Gulf Area

\begin{tabular}{|c|c|c|c|c|}
\hline Author/Year & Region & HPV Detection Method & Number /Type of Study & $\begin{array}{l}\text { HPV Positive/ HPV Genotypes } \\
\text { /Co infection }\end{array}$ \\
\hline $\begin{array}{l}\text { Raza et al., } 2010 \\
{[36]}\end{array}$ & Pakistn & $\begin{array}{l}\text { Home Brew PCR GP5/GP6 \& Reverse } \\
\text { Line Blot Hybridization }\end{array}$ & $\begin{array}{l}\text { 899/ Routine Checkups } \\
\text { \& Abnormal Cytology }\end{array}$ & $\begin{array}{l}2.78 \% \text { / HPV } \\
16,18,45,56,33,59 \text { / Not } \\
\text { Mentioned }\end{array}$ \\
\hline $\begin{array}{l}\text { Siddiqa et } \\
\text { al.,2014 [37] }\end{array}$ & Pakistn & $\begin{array}{l}\text { Home Brew PCR GP5/GP6 \& Duplex } \\
\text { PCR 16\&18 }\end{array}$ & 77/ Cervical Cancer & $\begin{array}{l}53.24 \% \text { / HPV16,18 / Not } \\
\text { Mentioned }\end{array}$ \\
\hline $\begin{array}{l}\text { Akbar et al., } \\
2015[38]\end{array}$ & Pakistn & $\begin{array}{l}\text { Home Brew PCR GP5/GP6 \& Duplex } \\
\text { PCR 16\&18 }\end{array}$ & 200/Routine Checkups & $\begin{array}{l}2 \% \text { / HPV 16,18 / Not } \\
\text { Mentioned }\end{array}$ \\
\hline $\begin{array}{l}\text { Shahid et al., } \\
2015 \text { [39] }\end{array}$ & Pakistn & $\begin{array}{l}\text { Home Brew PCR GP5/GP6 \& Duplex } \\
\text { PCR 16\&18 }\end{array}$ & 160/Routine Checkups & $\begin{array}{l}1.87 \% \text { / HPV16 / Not } \\
\text { Mentioned }\end{array}$ \\
\hline $\begin{array}{l}\text { Darnel et al., } \\
2010[40]\end{array}$ & Syria & Home Brew Specific PCR (E7) & $\begin{array}{l}\text { 44/ FFPE Cervical } \\
\text { Cancer }\end{array}$ & $\begin{array}{l}95.45 \% / \mathrm{HPV} \\
33,16,18,45,52,58,35,51,31 / \\
\text { Not Mentioned }\end{array}$ \\
\hline $\begin{array}{l}\text { Banna et al., } \\
2014[41]\end{array}$ & UAE & $\begin{array}{l}\text { Home Brew\& mix of } 13 \text { primers } \\
16,18,31,33,35,39,45,51,52,56,58,59,68\end{array}$ & 50/ Neg. Pap Smear Tests & $\begin{array}{l}\text { 10\% / Any of } 13 \text { types / Not } \\
\text { Mentioned }\end{array}$ \\
\hline $\begin{array}{l}\text { Bensumaidea et } \\
\text { al.,2014 [42] }\end{array}$ & Yemen & $\begin{array}{l}\text { Immunohistochemistry \& HPV16/18 kit } \\
\text { ( Sacace-Biotechnologies) }\end{array}$ & 84/ Cervical Cancer & $\begin{array}{l}\text { 100\% / HPV 16,18 / Not } \\
\text { Mentioned }\end{array}$ \\
\hline $\begin{array}{l}\text { Ahmed et al., } \\
2015 \\
{[43]}\end{array}$ & Yemen & $\begin{array}{l}\text { HPV 31,33,35,39,45 } \\
\text { ( Sacace-Biotechnologies) }\end{array}$ & $\begin{array}{l}\text { 200/ FFPE Cervial } \\
\text { Cancer \& Benign Tissue }\end{array}$ & 24\%/HPV 31,45,33,35,39/1\% \\
\hline $\begin{array}{l}\text { Haiija et } \\
\text { al.,2006 [44] }\end{array}$ & Bahran & Home Brew PCR \& RFLP & 100/ Routine Checkups & $\begin{array}{l}11 \% \text { / HPV 16,18,45,62,53 / } \\
\text { Not mentioned }\end{array}$ \\
\hline $\begin{array}{l}\text { Moosa et } \\
\text { al.,2014 [45] }\end{array}$ & Bahran & LiPA & 571/ Routine Checkups & $\begin{array}{l}9.8 \% \text { / } \\
\text { HPV52,16,31,51,6,70,74 / } \\
2.62 \%\end{array}$ \\
\hline $\begin{array}{l}\text { Fadhil et al., } \\
2014 \text { [46] }\end{array}$ & Iraq & $\begin{array}{l}\text { Genoaaray primer-based PCR } \\
21 \mathrm{HR} \& \text { LR types }\end{array}$ & 188/ Cervical Dysplasia & $\begin{array}{l}99.91 \% \text { ( } 82.35 \% \text { HR-types \& } \\
17.56 \% \text { LR types) / } 27.94 \%\end{array}$ \\
\hline $\begin{array}{l}\text { Al-Awadhi et } \\
\text { al., 2011 [47] }\end{array}$ & Kuwait & $\begin{array}{l}\text { Home Brew PCR GP5/GP6, } \\
\text { MY09/MY11 \& Sequencing }\end{array}$ & 3011/ Routine Checkups & $\begin{array}{l}\text { 2.3\% / HPV11,81,61,53,54, } \\
\text { 90,31,73,84,106, } \\
\text { 33,56,58,67,70,82,83,10 / Not } \\
\text { Mentioned }\end{array}$ \\
\hline $\begin{array}{l}\text { Al-Tahani et } \\
\text { al., } 2010[48]\end{array}$ & Qatar & $\begin{array}{l}\text { RT-PCR (E1-E2 region) } \\
\text { Sacace Biotechnologies, Italy }\end{array}$ & $\begin{array}{l}95 \% \text { / Abnormal } \\
\text { Screening }\end{array}$ & $64 \%$ / HPV 16,52,56/34.4\% \\
\hline $\begin{array}{l}\text { Elmi et al., } \\
2012[49]\end{array}$ & Qatar & $\begin{array}{l}\text { Real Time PCR } \\
\text { Sacace Biotechnology, Italy }\end{array}$ & 1100/ Routine Checkups & $\begin{array}{l}\text { 11.36\%/15 HPV Genotypes } \\
\text { Detected/ Not Mentioned }\end{array}$ \\
\hline $\begin{array}{l}\text { Bansal et al., } \\
2014[50]\end{array}$ & Qatar & $\begin{array}{l}\text { Real Time PCR } \\
\text { Sacace Biotechnology, Italy }\end{array}$ & 3008/ Routine Checkups & $\begin{array}{l}11.8 \% \text { / HPV 81,11,16,18,56/ } \\
0.93 \%\end{array}$ \\
\hline $\begin{array}{l}\text { Al-Sbeih et al., } \\
2011[51]\end{array}$ & Saudi Arabia & $\begin{array}{l}\text { Linear Array Detection Kit (LA DK) } \\
\text { (Roche) ( } 37 \text { genotypes) }\end{array}$ & 100/ Cervical Cancer & $\begin{array}{l}89 \% \text { / HPV } \\
16,18,31,39,45,51,59,73,6,64,7 \\
0 / 5.5 \%\end{array}$ \\
\hline $\begin{array}{l}\text { AlBadawi et } \\
\text { al.,2011 } \\
{[52]}\end{array}$ & Saudi Arabia & $\begin{array}{l}\text { Home Brew PCR GP5/GP6, } \\
\text { MY09/MY11 \& Sequencing \& Reverse } \\
\text { Line Blot Hybr (RLB) }\end{array}$ & 90/ Cervical Cancer & $\begin{array}{l}95.55 \% / \mathrm{HPV} \\
16,18,45,33,31,52,53,58,59,66 \\
/ 18.88 \%\end{array}$ \\
\hline $\begin{array}{l}\text { Al-Sbeih et al., } \\
2013 \\
{[53]}\end{array}$ & Saudi Arabia & $\begin{array}{l}\text { Linear Array HPV Genotyping } \\
\text { (Roche) ( } 37 \text { genotypes) }\end{array}$ & 318/ Cervical Cancer & $\begin{array}{l}82 \% / \mathrm{HPV} \\
16,31,18,45,73,32,70,592,59 / \\
2.2 \%\end{array}$ \\
\hline $\begin{array}{l}\text { Bondji et al., } \\
2013 \text { [54] }\end{array}$ & Saudi Arabia & Hybrid Capture II & 485/ Routine Checkups & $5.6 \%$ / Not Mentioned \\
\hline $\begin{array}{l}\text { Turki et al., } \\
2013[55]\end{array}$ & Saudi Arabia & $\begin{array}{l}\text { Multiplex PCR Plus BiofilmChip } \\
\text { Hybridization Assay ( } 26 \text { types) }\end{array}$ & 40/ Abnormal Screening & $42.5 \%$ / HPV 16,18,45 / 0\% \\
\hline $\begin{array}{l}\text { Al-Obaid et al., } \\
2014[56]\end{array}$ & Saudi Arabia & LiPA & 417/ Routine Checkups & $\begin{array}{l}6.95 \% / \\
\text { HPV68/73,18,16,31,51,52,39,5 } \\
6,58,6,42,54,11,40,70,74 \\
/ 0.95 \%\end{array}$ \\
\hline $\begin{array}{l}\text { Al-Ahdal et al., } \\
2014 \text { [57] }\end{array}$ & Saudi Arabia & Reverse Line Blot Hybridization & $\begin{array}{l}\text { 519/ Routine Checkups } \\
\text { \& Abnormal Cytology }\end{array}$ & $\begin{array}{l}31.6 \% \text { / HPV16,18,11/ Not } \\
\text { Mentioned }\end{array}$ \\
\hline
\end{tabular}

\section{Discussion}

WHO/International Information Center on HPV (ICO) and cervical cancer reports that more than 99\% of cervical cancers are associated with HPV infection (3). An organized prevention program is frequently reported to be needed for reducing the rate of cervical cancer in low and middle income countries (56). It is frequently reported that considerable number of females diagnosed with cervical cancer have never been screened by HPV testing or they may even be asymptomatic patients (39,58-60). Human Papillomavirus infection is diagnosed mainly by molecular methods and can be either DNA-based or RNA assays. The DNA-based assays are the best method for HPV detection and typing, especially the early stages of infection since 
their sensitivity are relatively higher than RNA based methods. However, once the disease has progressed, RNA-based assays are considered more accurate and recommended for cervical cancer infections. Human Papillomavirus DNA testing has been useful in decreasing mortality rates, which are due to cervical cancer up to $50 \%$ in developed countries (61-63). The usefulness, specificity, sensitivity and cost effectiveness of HPV DNA testing and genotyping have been compared to visual inspection methods for cervical cancer screening, which are reported to achieve the maximum effectiveness for patients, who are between 35 to 50 years old (62-64). An appropriate technique for use in clinical laboratory depends on parameters such as the cost of the assay, population to whom this test is to be applied, as well as specificity and sensitivity of the test. However, in under developed countries, cost effectiveness may be the most important factor. Human Papillomavirus genotyping methods merit attention in several aspects. Polymerase Chain Reaction hybridization and conventional amplification-based approaches have been used for HPV screening and genotyping. Each of these methods has some drawbacks, mainly in the area of reagents, cost effectiveness, time required, and false positive and negative results, especially in samples with lower copy numbers and mixed infections. Results of some surveys show that primers such as MY09/11 are ineffective due to increased mutations in HPV genome and multiple infections (65-66). Therefore, conventional PCR and other molecular methods should be improved in order to cover these new mutant types or to revise the screening methods by implementing other recommended molecular techniques for HPV detection and typing $(61,65,66)$. At present, valid genotyping procedures available commercially and in-house methods are approved in WHO proficiency testing, and could be considered as the best practical way for primary HPV testing in cervical cancer screening plans $(10,66)$. The most important problems of HPV screening procedures, which need to be considered, are false positive and negative results, sampling preparation and shipment specimens. However, the most important objective is HPV diagnosis in early stages of cervical cancer and precancerous lesions and other related cancers and infections (67-71). Unfortunately, our search showed no special attention on the majority of home brew methods to optimize the test in applied conditions, regarding detection limit, frequency, and false negative and positive results, except a few studies (12). Another missed point in these papers was ignoring HPV multiple infections that were underlined in reports applying standard and approved methods (4, 72-78). We did not expect a high rate of multiple infections in papers on normal populations attending gynaecology clinics, since it can be varied in each population. However, patients with cervical cancer or high-risk groups are at increased risk of infection with more than one type. The released data also showed that proper screening programs were almost missing to deprive females for early detection of cytological changes. The WHO reports on human papillomavirus and related cancers in Iran and its neighbouring countries showed that no comprehensive data are available from cancer registry on the incidence of cervical cancer, or age standardized incidence rate by histological types among females in this region (77).

Looking through these investigations and reported articles revealed some gaps, and lack of HPV strategy in policy makers of cervical cancer screening. Therefore, it seems that there is no HPV DNA primary testing plan for patients with cervical cancer in the countries of this region. This may be due to cost of the tests or lack of diagnostic surveillance system algorithm. Hence, the following suggestions can help the authorities and national policy-makers to overcome cancers related to HPV rate:

1. Commercializing the most popular molecular protocols in both screening and genotyping methods in order to reduce the sampling cost per test for health services and clinical laboratories.

2. Simultaneously performing co-testing of pap tests with molecular HPV testing for any screening of HPV infections.

3. Defining and designing an algorithm for care HPV, screening and prophylactic of cervical cancer programs. The designed algorithm can be different for any country, depending on the prevalence of most carcinogenic genotypes, environmental conditions and other parameters. 


\section{Conclusion}

Further planning for regular HPV screening and genotyping by authoritative methods and development of favorable national vaccination program is necessary to prevent incidence of cervical carcinoma in the future.

\section{Conflict of Interests}

The authors declare that there was no conflict of interest.

\section{References}

1. Shayanfar N, Hosseini N, Panahi M, Azadmanesh K, Mohammadpour M, Kadivar M, et al. Detection of mucosal type human papillomavirus in cutaneous squamous cell carcinoma in Iran. Pathol Res Pract 2013; 209: 90-94.

2. Sohrabi A, Mirab-Samiee S, Rahnmaye-Farzami M, Rafizadeh M, Akhavan S, Hashemi-Bahremani M, et al. C13orf18 and C1orf166 (MULAN) DNA genes methylation are not associated with cervical cancer and precancerous lesions of human papillomavirus genotypes in Iranian women. Asian Pac J Cancer Prev 2014; 15: 6745-6748.

3. Human Papillomavirus Laboratory Manual 2009. Available at: http:// www.who.int/vaccines-documents. Accessed: November 2010.

4. Establishing the performance characteristics of in vitro diagnostic devices for the detection or detection and differentiation of human papillomaviruses. Available at: http://www.fda.gov/ downloads/medicaldevices/ deviceregulationandguidance/guidancedocuments/ucm4 58179.pdf. Accessed: August 2015.

5. Al-Awadhi R, Chehadeh W, Kapila K. Prevalence of human papillomavirus among women with normal cervical cytology in Kuwait. J Med Virol 2011; 83(3): 453-460.

6. Sohrabi A, Rahnmaye-Farzami M, Mirab-Samiee S, Mahdavi S, Babei M. Comparison of In-House Multiplex Real Time PCR, DiagcorGenoFlow HPV Array test and INNO-LiPA HPV Genotyping Extra Assays with LCD- Array kit for human papillomavirus genotyping in cervical liquid based cytology specimens and genital lesions in Tehran, Iran. Clin Lab 2016; 62 (4): 615-619.

7. Abru ALP, Souza RP, Gimenes F, Consolaro MEL. A review of methods for detect human Papillomavirus infection. Virol J 2012; 9:262.

8. Ashley A, Bennett KM. Molecular diagnostics of human papillomavirus. Lab Med 2010; 41: 523-530.

9. Tornesello ML, Buonaguro L, Giorgi-Rossi P, Buonaguro FM. Viral and cellular biomarkers in the diagnosis of cervical intraepithelial neoplasia and cancer. Biomed Res Int 2013; 2013: 519619.

10. Iftner $\mathrm{T}$, Germ L, Swoyer R, Kjaer SK, Breugelmans JG, Munk C, et al. Study comparing human papillomavirus (HPV) real-time multiplex PCR and Hybrid Capture II INNO-LiPA v2 HPV genotyping PCR assays. J Clin Microbiol 2009; 47(7): 2106-2113.

11. Nielsen A, Iftner T, Nørgaard M, Munk C, Junge J, Kjaer SK. The importance of low-risk HPV infection for the risk of abnormal cervical cytology/histology in more than 40000 Danish women. Sex Transm Infect 2012; 88(8): 627-632.

12. Sohrabi A, Mirab-Samiee S, Modarresi MH, Izadimood N, Azadmanesh K, RahnmayeFarzamiM.Development of in-house multiplex real time PCR for human papillomavirus genotyping in iranian women with cervical cancer and cervical intraepithelial neoplasia. Asian Pac J Cancer Prev 2014; 15: 6257-6261.

13. Hamkar R, Mokhtari Azad T, Mahmoodi M, SeyediRashti SR, Yahyapour, Severini R, et al. Prevalence of various type of human papilloma virus among cervical cancer and normal biopsy in the Mazandaran Province in Iran. Iran J Infect Dis Trop Med 2003; 8 (22): 9-15.

14. Mortazavi S, Zali M, Raoufi M, Nadji M, Kowsarian P, NowrooziA. The prevalence of human papillomavirus in cervical cancer in Iran. Asian Pac J Cancer Prev 2002; 3(1): 69-72.

15. Hamkar R, Azad TM, Mahmoodi M, Seyedirashti S, Severini A, NateghR. Prevalence of human papillomavirus in Mazandaran Province, Islamic Republic of Iran. East Mediterr Health J 2002; 8(6): 805811.

16. Farjadian S, Asadi E, Doroudchi M, SamsamiDehaghani A, Tabei SZ, Kumar VP, et al. High Risk HPV types in Southern Iranian patients with cervical cancer. Pathol Oncol Res 2003; 9(2):121-125.

17. Zandi K, Eghbali SS, Hamkar R, Ahmadi S, Ramedani E, Deilami I, et al. Prevalence of various human papillomavirus (hpv) genotypes among women who subjected to routine pap smear test in bushehr city (south west of Iran) 2008-2009. Virol J 2010; 7:65.

18. Safaei A, Khanlari M, Momtahen M, Monabati A, Robati M, Amooei S, et al. Prevalence of high-risk human papillomavirus types 16 and 18 in healthy women with cytologically negative pap smear in Iran. Indian $\mathbf{J}$ Pathol Microbiol 2010; 53(4): 681-685.

19. Shahramian I, Heidari Z, Mahmoudzadeh-Sagheb H, Moradi A, Forghani F. Prevalence of HPV infection and high risk HPV genotypes (16, 18), among monogamous and polygamous women, in Zabol, Iran. Iran J Public Health 2011; 40(3):113-121.

20. Jaberipour M, Samsami A, Sahraiian F, Kazerooni T, Hashemi M, Ghaderi A, et al. Elevation of 
HPV-18 and HPV-16 DNA in the plasma of patients with advanced cervical cancer. Asian Pac J Cancer Prev 2011; 12(1):163-167.

21. Jaberipour M, Momtahan M, Najib F, Amooei S, Saidifard F, Ghaderi A, et al. Detection of high-risk human papillomavirus types 16 and 18 but not 33 and 52 in external genital warts from Iranian females. Asian Pac J Cancer Prev 2011; 12(3):771-774.

22. Moradi A, BakhshandehNosrat S, Besharat S. Molecular epidemiology of highrisk types of human papillomaviruses $(16,18)$ in papsmear, the north east of Iran. Iran J Cancer Prev 2011; 3:135-140.

23. Allameh T, Moghim S, Asadi-Zeidabadi M. A survey on the prevalence of high-risk subtypes of human papilloma virus among women with cervical neoplasia in Isfahan University of medical science. Arch Gynecol Obstet 2011; 284(6):1509-13.

24. Shahsiah R, Khademalhosseini M, Mehrdad N, Ramezani F, NadjiiSA.Human papillomavirus genotypes in Iranian patients with crvical cancer. Pathol Res Pract 2011; 207(12):754-757.

25. Khodakarami N, Clifford GM, Yavari P, FarzanehF,Salehpour S, Broutet $\mathrm{N}$, et al.Human papillomavirus infection in women with and without cervical cancer in Tehran, Iran. Int J Cancer 2012; 131:E156-E161.

26. Eghbali SS, Amirinejad R, Obeidi N, Mosadeghzadeh S, Vahdat K, Azizi F, et al. Oncogenic human papillomavirus genital infection in Southern Iranian women: population-based study versus clinicbased data. Virol J 2012; 9:194.

27. Hamidi-Fard M, Fattahi-Abdizadeh M, Makvandi M, Ranjbari N, Mansoori E, SamarbafZadehAR.Detection and genotyping of human papillomavirus in cervical tissue samples in Ahvaz, Southwest Iran. Jundishapur J Microbiol 2013; 6(7): e4569.

28. Moeini Z, tahmasebiFard Z, Abdirad A, Seyed Ali Roteh S, SarmadiS.Invetigation of correlation between human papillomavirus and endometriosis lesion. NCMBJ 2012; 2(6):67-73.

29. Haghshenas M, Golini-Moghaddam T, Rafiei A, Emadeian O, Shykhpour A, Ashrafi GH. Prevalence and type distribution of high-risk human papillomavirus in patients with cervical cancer: a population-based study. Infect Agent Cancer 2013; 8(1):20.

30. Shafaghi B, Jarollahi A, Yousefzadeh B, Ameri A, Moghadam S, MostafaviM.Human papillomavirus prevalence and types among Iranian women attending regular gynecological visit. Rep Radiother Oncol 2013; 1(2): 73-79.

31. MobiniKeshe M, Kafashi A, BagheriGh, Shahkarami MK, Mohammadi M, Nadji SA. Identification of human papillomavirus type 16 among thinprep samples from 11 provinces of Iran. Iran J Obstet Gynecology Infertil 2013; 16(72): 22-28.

32. Shanehsazzadeh M, Sharifi-Rad J, Behbahani M, PourazarA.Analysis of human papillomavirus and herpes simplex virus genus -2 from patients with cervical cancer in Isfahan, Iran. Mater Sociomed 2014; 26(4): 234-236.

33. Pouryasin M, Sharafi H, Mousavi AS, Khodadad S, Marjani M, Jamshidi F, et al. Distribution of human papillomavirus genotypes in liquid-based samples; abundance of HPV-53 in Tehran, Iran. Iran J Public Health 2014; 43(8):1159-1160.

34. Mirzaie-Kashani E, Bouzari M, Talebi A, Arbabzadeh-Zavareh F. Detection of human papillomavirus in chronic cervicitis, cervical adenocarcinoma, intraepithelial neoplasia and squamus cell carcinoma. Jundishapur J Microbiol 2014; 7(5): e9930.

35. Mehran SM, Ghanaei MM, Mojtehadi A. The prevalence of human papilloma virus (HPV) in women using liquid base pap smear in Rasht, Northern of Iran. J Clin Diagn Res 2015; 9(7): IC01-IC02.

36. Raza SA, Franceschi S, Pallardy S, Malik FR, Avan BI, Zafar A, et al. Human papillomavirus infection in women with and without cervical cancer in Karachi, Pakistan. Br J Cancer 2010; 102: 1657-1660.

37. Siddiqa A, Zainab M, Qadri I, Bhatti MF, Parish JL.Prevalence and genotyping of high risk human papillomavirus in cervical cancer samples from Punjab, Pakistan. Viruses 2014; 6(7): 2762-2777.

38. Akbar S, Pervez SN, Shah W. Manual liquid based cytology for Pap smear preparation and HPV detection by PCR in Pakistan.Asian Pac J Cancer Prev 2015; 16(2):579-583.

39. Shahid M, Kazmi SU, Rehman A, Ainuddin J, Furqan S, Nazeer S.Cervical cancer screening and HPV genotype distribution among asymptomatic patients of Karachi Pakistan. Pak J Med Sci 2015; 31(3): 493-498.

40. Darnel AD, Wang D, Ghabreau L, Yasmeen A, Sami S, Akil N, et al. Correlation between the presence of high-risk human papillomaviruses and Id gene expression in Syrian women with cervical cancer. Clin Microbiol Infect 2010; 16(3): 262-266.

41. Banna NE, Ghaith Al, Eyd GA, Saeed RS. Highrisk human papillomavirus infection among women with pap smear tests negative for intraepithelial lesions or malignancy. Int J Med 2014; 4(1):102-106.

42. Bensumaidea SH, Ahmed HG, Bafakeer SS, Abdal-Aziz MS, Alshammari FD. A correlation of immunohistochemical and molecular detection of human papillomavirus subtypes 16 and 18. Int J Sci Res 2014; 3(11):2487-2490.

43. Ahmed HG, Bensumaidea SH, Ashankyty IM. Frequency of Human Papilloma Virus (HPV) subtypes 
$31,33,35,39$ and 45 among Yemeni women with cervical cancer. Infect Agent Cancer 2015; 10:29.

44. Hajjaj AA, Senok AC, Al-Mahmeed AE, Isaa AA, Arzese AR, BottaGA. Human papillomavirus infection among women attending health facilities in the Kingdom of Bahrain. Saudi Med J 2006; 27:487-491.

45. Moosa K, Alsayyad AS, Quint W, Gopala K, DeAntonioR.An epidemiological study assessing the prevalence of human papillomavirus types in women in the Kingdom of Bahrain. BMC Cancer 2014; 14:905.

46. Fadhil HY, Saleh DS, Al-Hamdani FG. Detection of human papillomavirus 21 genotypes in a sample of Iraqi women with cervical abnormalities and cancer. Can J Pure Appl Sci 2014; 8(1): 2671-2676.

47. Awadhi R, Chehadeh W, Al-Jassar W, Al-Harmi J, Al-Saleh E, Kapila K. Viral load of human papillomavirus in women with normal and abnormal cervical cytology in Kuwait. J Infect Dev Ctries 2013; 7: 130-136.

48. Al-Thani A, Abu-Rub A, Al-Ansari A, Abushama M, Al-Khanji M, Al-Lawati S. Prevalence of human Papillomavirus infection in women attending gynecology /oncology clinic in Qatar. Future Virol 2010; 5:513-519.

49. Elmi A, Bansal D, Al-Thani A, Al-Ansari A, Mohamed N, Sultan A. Molecular epidemiology of human papillomavirus among Arab women in Qatar. Available at http://www.qscience.com /doi/abs/10.5339 /qfarf.2012.BMP6. Accessed: 2012.

50. Bansal D, Elmi AA, Skariah S, Haddad P, AbuRaddad LJ, Al Hamadi AH, et al. Molecular epidemiology and genotype distribution of Human Papillomavirus (HPV) among Arab women in the State of Qatar. J Transl Med 2014; 12:300.

51. Alsbeih G, Ahmed R, Al-Harbi N, Venturina LA, Tulbah A, Balaraj K. Prevalence and genotypes' distribution of human papillomavirus in invasive cervical cancer in Saudi Arabia. Gynecol Oncol 2011; 121(3):522-526.

52. Al-Badawi IA, Al-Suwaine A, Al-Aker M, Asaad L, Alaidan A, Tulbah A, et al. Detection and genotyping of human papiloma virus in cervical cancer specimens from Saudi patients. Int J Gynecol Cancer 2011;21: 907910.

53. Alsbeih G, Al-Harbi N, El-Sebaie M, Al-Badawi I. HPV prevalence and genetic predisposition to cervical cancer in Saudi Arabia. Infect Agent Cancer 2013;8(1):15

54. Bondagji NS, Gazzaz FS, Sait K, Abdullah L. Prevalence of high-risk human papillomavirus infections in healthy Saudi women attending gynecologic clinics in the western region of Saudi Arabia. Ann Saudi Med 2013;33: 13-17.

55. Turki R, Sait K, Anfinan N, Sohrab SS, AbuzenadahAM.Prevalence of human papillomavirus in women from Saudi Arabia. Asian Pac J Cancer Prev 2013; 14: 3177-3181.

56. Al-Obaid A, Al-Badawi I, Al-Kadri H, Gopala K, Kandeil W, Quint W, et al. Human papillomavirus prevalence and type distribution among women attending routine gynecological examinations in Saudi Arabia. BMC Infect Dis 2014; 14: 643.

57. Al-Ahdal MN, Al-Arnous WK, Bohol MF, Abuzaid SM, Shoukri MM, Elrady KS, et al. Human papillomaviruses in cervical specimens of women residing in Riyadh, Saudi Arabia: a hospital-based study. J Infect Dev Ctries2014;8: 320-325.

58. Khorasanizadeh F, Hassanloo J, Khaksar N, Taheri SM, Marzaban M, Rashidi BH, et al. Epidemiology of cervical cancer and human papilloma virus infection among Iranian women - analyses of national data and systematic review of the literature. Gynecol Oncol 2012;128: 277-281.

59. Nishino HT, Tambouret RH, Wilbur DC. Testing for human papillomavirus in cervical cancer screening: a review of indications and methodology. Cancer Cytopathol 2011; 119(4): 219-227.

60. Evans W, Filippova M, Swensen R. Modern molecular and clinical approaches to eradicate hpvmediated cervical cancer. Available at http://cdn.intechopen.com/pdfs-wm/44375.pdf. Accessed: 2012.

61. Cuzick J, Arbyn M, Sankaranarayanan R, Tsu V, Ronco G, Mayrand MH, et al. Overview of human papillomavirus-based and other novel options for cervical cancer screening in developed and developing countries. Vaccine 2008; 19; 26Suppl 10:K29-41.

62. Meijer CJ, Berkhof J, Castle PE, Hesselink AT, Franco EL, Ronco G, et al. Guidelines for human papillomavirus DNA test requirements for primary cervical cancer screening in women 30 years and older. Int J Cancer 2009; 124(3):516-520.

63. Benevolo M, Vocaturo A, Caraceni D, French D, Rosini S, Zappacosta R, et al. Sensitivity, Specificity, and Clinical Value of Human Papillomavirus (HPV) E6/E7 mRNA Assay as a Triage Test for Cervical Cytology and HPV DNA Test. J Clin Microbiol 2011; 49(7): 26432650 .

64. Shi JF, Canfell K, Lew JB, Qiao YL. The burden of cervical cancer in China: synthesis of the evidence. Int J Cancer 2012; 130 (3):641-52.

65. Şahiner F, Kubar A, Gümral R, Ardıç M, Yiğit N, Şener K, et al. Efficiency of MY09/11 consensus PCR in the detection of multiple HPV infections. Diagn Microbiol Infect Dis 2014 Sep; 80(1):43-49.

66. Torres M, Fraile L, Echevarria J, Hernandez Novoa B, Ortiz M. Human papillomavirus (HPV) genotyping: automation and application in routine laboratory testing. Open Virol J 2012; 6:144-50. 
67. Schmitt M, Dondog B, Waterboer T, Pawlita M, Tommasino M, Abundance F.Abundanceof Multiple highrisk human papillomavirus (HPV) infections found in cervical cell analyzed by use of an ultrasensitive HPV genotypingassay. J Clin Microbiol 2010; 48(1):143-9.

68. Hajia M, Safadel N, Samiee SM, Dahim P, Anjarani S, Nafisi N, et al. Quality Assurance Program for Molecular Medicine Laboratories. Iran J Pub Health 2013; 42 Supple1:119-124.

69. Yin D, Jiang Y, Wang N, Ouyang L, Lu Y, Zhang $\mathrm{Y}$, et al. The diagnosticvalue of serum hybrid capture 2 (HC2) HPV DNA in cervical cancer: a systematic review andmeta-analysis. Tumor Biol 2014; 35(9):9247-53.

70. Shayanfar N, Babaheidarian P, Rahmani H, Azadmanesh K, Sohrabi A, MohammadpourM,et al. EpidermodysplasiaVerruciformis Associated with Plasmablastic Lymphoma and Hepatitis B Virus Infection. Acta Dermatovenerol Croat 2012; 20(4):267271.

71. Sohrabi A, RahnamayeFarzami, MirabSamiee S, ModarressiMH.An overview on papillomaviruses as the main cause of cervical cancer. Iran J Obstet Gynecology Infertil 2015; 18(145):14-25.

72. Lebelo RL, Bogers JJ, Thys S, Depuydt C, Benoy I, Selabe SG, et al. Detection, genotyping and quantitation of multiple HPV infections in South African women with cervicalsquamous cell carcinoma. J Med Virol 2015; 87(9): 1594-600.
73. Shi JF, Canfell K, Lew JB, Zhao FH, Legood R, Ning Y, et al.Evaluation of primary HPV-DNA testing in relation to visual inspection methods for cervical cancer screening inrural China: an epidemiologic and costeffectiveness modelling study. BMC Cancer 2011; 11:239.

74. Herrero R, Castle PE, Schiffman M, Brattil MC, Hildesheim A, Morales J, et al. Epidemiologic profile of typespecific human papillomavirus infection and cervical neoplasia in Guanacaste, Costa Rica. J Infect Dis 2005; 191:1796-807.

75. Wentzensen N, Schiffman M, Dunn T, Zuna RE, Gold MA, Allen RA, et al. Multiple human papillomavirus genotype infections in cervical cancer progression in the study to understand cervical cancer early endpoints and determinants. Int J Cancer 2009; 125:2151-8.

76. Trottier H, Mahmud S, Costa MC, Sobrinho JP, Duarte-Franco E, Rohan TE, et al. Human papillomavirus infections with multiple types and risk of cervical neoplasia. Cancer Epidemiol Biomarkers Prev 2006; 15:1274-80.

77. WHO/ICO HPV Information Centre. Human papillomavirus and related cancers, specific Iran summary report.Available athttp://www.hpvcentre.net/ summaryreport.php.Accessed: Mar 2015.

78. Denny L, Prendiville W. Cancer of the cervix: Early detection and cost-effective solutions. Int $\mathrm{J}$ Gynaecol Obstet 2015; 131 Suppl 1:S28-32.

\section{How to Cite This Article:}

Sohrabi A, Hajia M. Cervical Cancer and Genital Infections: Assessment of Performance and Validation in Human Papillomavirus Genotyping Assays in Iran, its Neighbouring Countries and Persian Gulf Area. Iranian Journal of Pathology. 2017; 12(1): 35-44. 